\title{
Computability of continuous solutions of higher-type equations
}

\author{
Martín Escardó \\ School of Computer Science, University of Birmingham, UK
}

Version of October 31, 2009

\begin{abstract}
Given a continuous functional $f: X \rightarrow Y$ and $y \in Y$, we wish to compute $x \in X$ such that $f(x)=y$, if such an $x$ exists. We show that if $x$ is unique and $X$ and $Y$ are subspaces of Kleene-Kreisel spaces of continuous functionals with $X$ exhaustible, then $x$ is computable uniformly in $f, y$ and the exhaustion functional $\forall_{X}: 2^{X} \rightarrow 2$. We also establish a version of the above for computational metric spaces $X$ and $Y$, where is $X$ computationally complete and has an exhaustible set of Kleene-Kreisel representatives. Examples of interest include functionals defined on compact spaces $X$ of analytic functions. Our development includes a discussion of the generality of our constructions, bringing QCB spaces into the picture, in addition to general topological considerations.
\end{abstract}

Keywords and phrases. Higher-type computability, Kleene-Kreisel spaces of continuous functionals, exhaustible set, searchable set, QCB space, admissible representation, topology in the theory of computation with infinite objects.

\section{Introduction}

Given a continuous functional $f: X \rightarrow Y$ and $y \in Y$, we consider the equation $f(x)=y$ with the unknown $x \in X$. We show that if $X$ and $Y$ are subspaces of Kleene-Kreisel spaces [8] with $X$ exhaustible [6], the solution is computable uniformly in $f, y$ and the exhaustion functional $\forall_{X}: 2^{X} \rightarrow 2$, provided it is unique (Section 47). Moreover, under the same assumptions for $X$ and $Y$, it is uniformly semi-decidable whether a solution $x \in X$ fails to exist.

Similar results are known in constructive mathematics [4], but with a different level of generality and different foundational assumptions. Here exhaustibility plays the role of a computational counter-part of the topological notion of compactness (Section 3). Recall that the Kleene-Kreisel spaces are obtained from the discrete space $\mathbb{N}$ by iterating finite products and function spaces in a suitable cartesian closed category (e.g. filter spaces, limit spaces, $k$-spaces, equilogical spaces or QCB spaces).

The computation of unique solutions of equations of the form $g(x)=h(x)$ with $g, h: X \rightarrow Y$ is easily reduced to the previous case, because there are (abelian) computable group structures on the ground types that can be lifted componentwise to product types and pointwise to function types, and hence $x \in X$ is a solution of such an equation iff it is a solution of the equation $f(x)=0$, where $f(x)=h(x)-g(x)$. And, by cartesian closedness, the case in which $g$ and $h$ computably depend on parameters, and in which the solution computably depends on the same parameters, is covered. Moreover, because the Kleene-Kreisel spaces are closed under finite products and countable powers, this includes the solution of finite and countably infinite systems of equations with functionals of finitely many or countably infinitely many variables. 
We also consider a generalization to computational metric spaces that applies to computational analysis, where $f$ can be a functional and $x$ a function (Section 5). And, as an application, we develop examples of sets of analytic functions that are exhaustible and can play the role of the space $X$ (Section 6 ).

Background. Our main reference to computation over Kleene-Kreisel spaces of continuous functionals is Normann [8] (see also his expository and survey papers [9, 10]). The Kleene-Kreisel spaces can be constructed in a variety of ways, some of them alluded above. There are also a number of equivalent approaches to computation over such spaces, based e.g. on total elements of effectively given domains or on Kleene associates (which amount to admissible representations in the sense of Weihrauch [13, 11]). In every such approach, one has a cartesian closed category, and hence computable maps are closed under $\lambda$-definability, which is the tool we apply to establish computability results. We reduce non-computability results to failure of continuity. In view of the previous two sentences, it is not strictly necessary to be acquainted with the technical notions and results in the form presented in the above references in order to be able to rigorously follow our presentation, provided the reader has some familiarity with the $\lambda$-calculus and with continuity at higher types. See e.g. [7] regarding continuity and other topological properties. We often rely on the density theorem [5], in the form that says that the Kleene-Kreisel spaces have computable dense sequences. We also use the fact that every Kleene-Kreisel space is a computable retract of a space of the form $\mathbb{N}^{X}$ with $X$ a Kleene-Kreisel space.

Generality of the underlying theory for our results. In fact, most of our results hold for all such retracts, and sometimes even more generally, as can be seen directly from the proofs we offer. The right setting for our presentation, in terms of generality, seems to be the category of effectively presented QCB spaces [1], where by an effective presentation we mean an effectively admissible representation in the sense of Schröder [11] (see the discussion of Section 8 of [1]).

Organization. Section (2): Discussion of the assumptions for our main results. The assumptions are familiar from constructive mathematics, but the discussion is intended to shed some light in the computational developement of later sections.

Section (3): Exhaustible spaces. This amounts to background, but it also includes new observations regarding previous work.

Section (4): Equations over Kleene-Kreisel spaces. This and the next section formulate and develop the main results of this this paper.

Section (5): Equations over metric spaces.

Section (6): Exhaustible spaces of analytic functions. This develops some applications.

\section{Preliminary discussion}

We begin by justifying the technical assumptions made in later sections and that were briefly mentioned in the introduction. Given a continuous function $f: X \rightarrow Y$ of KleeneKreisel spaces, or more generally of computable retracts of Kleene-Kreisel spaces, and a point $y \in Y$, we wish to compute a point $x \in X$, uniformly in $f$ and $y$, such that

$$
f(x)=y
$$

We emphasize that, in this paper, including Section 5 on metric spaces, the terminology uniform is used in the sense of recursion theory, rather than metric topology (the notions of uniform continuity and uniform convergence don't feature in this paper). 
We discuss several cases for $X$ and $Y$ and explain why, in general, further assumptions and data are required in general. The simplest case is $X=Y=\mathbb{N}$, for which the algorithm

$$
\mu x . f(x)=y
$$

computes a solution if and only if a solution exists. This is subsumed by the next case.

Consider $X$ arbitrary and $Y=\mathbb{N}$. By the Kleene-Kreisel density theorem, $X$ has a computable dense sequence $\delta: \mathbb{N} \rightarrow X$, and, by continuity of $f$ and discreteness of $\mathbb{N}$, if the equation has a solution, there is one of the form $x=\delta_{n}$ for some $n$. Hence the algorithm

$$
x=\delta_{\mu n . f\left(\delta_{n}\right)=y}
$$

computes a solution if and only if a solution exists. Moreover, in this particular case it is semi-decidable whether a solution exists, with the algorithm $\exists$ n. $f\left(\delta_{n}\right)=y$. Notice also that, by density of $\delta$ and discreteness of $\mathbb{N}$, whenever there is a non-isolated solution, there are uncountably many solutions unless $X=\mathbb{N}$, and so solutions are never unique if $X$ is a Kleene-Kreisel space distinct from $\mathbb{N}$.

Now consider $X=2$ and $Y=\mathbb{N}^{\mathbb{N}}$. Then a function $f: X \rightarrow Y$ amounts to two functions $f_{0}, f_{1}: \mathbb{N} \rightarrow \mathbb{N}$, and computing a solution to the above equation amounts to finding $i \in 2$ such that $f_{i}=y$, that is, $f_{i}(n)=y(n)$ for all $n \in \mathbb{N}$. In other words, under the assumption that

$$
f_{0}=y \text { or } f_{1}=y,
$$

we want to find $i$ such that $f_{i}=y$. If the only data supplied to the desired algorithm are $f_{0}, f_{1}, y$, this is not possible, because no finite amount of information about the data can determine that one particular disjunct holds (a similar situation is worked out in detail below). However, if we instead assume that

one of $f_{0}=y$ and $f_{1}=y$ holds, but not both,

then we can compute $i$ as follows:

Find the least $n$ such that $f_{0}(n) \neq y(n)$ or $f_{1}(n) \neq y(n)$, and let $i$ be the unique number such that $f_{i}(n)=y(n)$.

Thus, in general, it is not possible to compute solutions unless we know that they are unique, and in this particular case one can compute unique solutions. This kind of phenomenon is well known in constructive mathematics - see e.g. [3].

Next consider $X=\mathbb{N}$ and $Y=\mathbb{N}^{\mathbb{N}}$, and assume that the equation $f(x)=y$ has a unique solution. Now it is no longer possible to compute it uniformly in $f$ and $y$. For suppose there existed a computable partial functional $s:\left(\mathbb{N}^{\mathbb{N}}\right)^{\mathbb{N}} \times \mathbb{N}^{\mathbb{N}} \rightarrow \mathbb{N}$, defined on some superset of

$$
S=\left\{(f, y) \mid f(x)=y \text { has a unique solution } x_{0}\right\},
$$

such that $x_{0}=s(f, y)$ is the solution for $f, y \in S$. By continuity, for any $(f, y) \in S$ there is a number $n$ such that $s(f, y)=x_{0}=s(g, y)$ for every $g$ such that $g(x)(i)=f(x)(i)$ for all $x<n$ and $i<n$. W.l.o.g. we can assume that $x_{0}<n$ by replacing $n$ by $\max \left(n, x_{0}\right)+1$ if necessary. Choose $g$ defined by

$$
g(x)(i)= \begin{cases}f(x)(i) & \text { if } x<n \text { and } i<n, \\ y(i) & \text { if } x=n \\ y(i)+1 & \text { otherwise }\end{cases}
$$

By construction, $x=n$ is the unique solution of $g(x)=y$, and hence $s(g, y)=n$, which contradicts $s(g, y)=x_{0}$, and concludes the proof of the impossibility claim. However, if we know that there is a unique solution in a finite set $K \subseteq \mathbb{N}$, then the solution can be found uniformly in $f, y$ and a finite enumeration $e_{0}, \ldots, e_{k-1}$ of $K$, as follows: 
Find $n$ and $j<k$ such that the decidable conditions $\forall i<n . f\left(e_{j}\right)(i)=y(i)$ and $\forall l<k, l \neq j . \exists i<n . f\left(e_{l}\right)(i) \neq y(i)$ hold, and take $x=e_{j}$.

This generalizes the situation $X=2$, and is a particular case of Theorem 4.1 below, which shows that unique solutions in exhaustible subsets $X$ of Kleene-Kreisel spaces are computable uniformly in $f, y$ and $\forall_{X}$.

\section{Exhaustible Spaces}

In previous work we investigated exhaustible sets of total elements of effectively given domains and their connections with Kleene-Kreisel spaces of continuous functionals [6]. Here we work directly with exhaustible subspaces of Kleene-Kreisel spaces, where in this section we import notions and results for them from that work. Denote by $Y^{X}$ the space of continuous functionals from $X$ to $Y$. For the sake of definiteness and generality of the definitions, we work in the ambient cartesian closed category of effectively presented QCB spaces, discussed in the introduction.

Definition 3.1. Let $2=\{0,1\}$ be discrete.

1. A space $K$ is called exhaustible if the universal quantification functional

$$
\forall_{K}: 2^{K} \rightarrow 2
$$

defined by $\forall_{K}(p)=1$ if and only if $p(x)=1$ for all $x \in K$ is computable.

2. It is called searchable if there is a computable selection functional

$$
\varepsilon_{K}: 2^{K} \rightarrow K
$$

such that for all $p \in 2^{K}$, if there is $x \in K$ with $p(x)=1$ then $p\left(\varepsilon_{K}(p)\right)=1$.

3. A set $F \subseteq X$ is decidable if its characteristic map $X \rightarrow 2$ is computable.

Equivalently, $K$ is exhaustible if and only if the functional $\exists_{K}: 2^{K} \rightarrow 2$ defined by $\exists_{K}(p)=1$ if and only if $p(x)=1$ for some $x \in K$ is computable. If $K$ is searchable, then it is exhaustible, because $\exists_{K}(p)=p\left(\varepsilon_{K}(p)\right)$. The empty space is exhaustible, but not searchable, because there is no map $2^{\emptyset} \rightarrow \emptyset$.

The following results are directly adapted to our setting from [6].

\section{Lemma 3.2.}

1. The Cantor space $2^{\mathbb{N}}$ is searchable.

2. Any exhaustible subspace of a Kleene-Kreisel space is compact, and moreover, if it is non-empty, it is searchable, a computable retract, and a computable image of the Cantor space.

3. Searchable spaces are closed under computable images, intersections with decidable sets, and finite products.

4. A product of countably many searchable subspaces of a common Kleene-Kreisel space is searchable uniformly in the sequence of quantifiers.

Thus, exhaustibility is seen to be a computational counter-part of the topological notion of compactness, at least for subspaces of Kleene-Kreisel spaces. 
Remark 3.3. There is a subtle and important difference between the setting considered here and that considered in [6], already discussed in Section 8.7 of [6]. This is because the definition of exhaustibility studied there is more general and stronger, for two reasons: (1) exhaustibility is defined for subsets of a given space, and (2) the relevant predicates need to be defined on the subsets but are not required to be total in the ambient space. For the weaker notion considered here, which is enough for our purposes, the fact that exhausible spaces are searchable is an easy consequence of the density theorem. In fact, if $K$ has a dense sequence $\delta: \mathbb{N} \rightarrow K$, then the definition $\varepsilon(p)=\delta_{\mu n . \exists(p)=p\left(\delta_{n}\right)}$ shows that exhaustibility implies searchability. The non-trivial insight of [6] is that an exhaustible subset of a Kleene-Kreisel space is a retract, which is needed to show that exhaustible sets are searchable under the stronger definitions. Part of this insight is reconstructed here in Theorem 4.2 below, which is a consequence of one of the two main theorems proved here, namely 4.1

\section{Equations over Kleene-Kreisel Spaces}

Theorem 4.1. If $f: X \rightarrow Y$ is a continuous map of subspaces of Kleene-Kreisel spaces with $X$ exhaustible, and $y \in Y$, then, uniformly in $\forall_{X}, f$, and $y$ :

1. It is semi-decidable whether the equation $f(x)=y$ fails to have a solution.

2. If $f(x)=y$ has a unique solution $x \in X$, then it is computable.

Hence if $f: X \rightarrow Y$ is a computable bijection then it has a computable inverse, uniformly in $\forall X$ and $f$.

The conclusion is a computational counter-part of the topological theorem that any continuous bijection from a compact Hausdorff space to a Hausdorff space is a homeomorphism. This gives an alternative route to the following fact established in [6]:

Theorem 4.2. Any exhaustible subspace of a Kleene-Kreisel space is computably homeomorphic to an exhaustible subspace of the Baire space $\mathbb{N}^{\mathbb{N}}$.

Proof. Let $K$ be an exhaustible $k k$-space, let $s: K \rightarrow \mathbb{N}^{Z}$ and $r: \mathbb{N}^{Z} \rightarrow K$ be computable maps with $r \circ s=\operatorname{id}_{K}$ and $Z$ a Kleene-Kreisel space, and let $\delta_{n} \in Z$ be a computable dense sequence. The subspace $X=s(K) \subseteq \mathbb{N}^{Z}$, being a computable image of an exhaustible space, is itself exhaustible. As in [6], we consider the map $X \rightarrow \mathbb{N}^{\mathbb{N}}$ that sends $u \in X$ to the sequence $u\left(\delta_{n}\right)$, but we argue using Theorem 4.1 instead. Let $f: X \rightarrow Y$ be the restriction of this map to its image $Y \subseteq \mathbb{N}^{\mathbb{N}}$. By density, $f$ is one-to-one, and, by construction, it is onto, and hence it has a computable inverse. Therefore there is computable map $g: K \rightarrow Y$ defined by $g(k)=f(s(k))$ with computable inverse given by $g^{-1}(\alpha)=r\left(f^{-1}(\alpha)\right)$.

The following will be applied to semi-decide absence of solutions:

Lemma 4.3. Let $X$ be an exhaustible subspace of a Kleene-Kreisel space and $K_{n} \subseteq X$ be a sequence of sets that are decidable uniformly in $n$ and satisfy $K_{n} \supseteq K_{n+1}$. Then, uniformly in the data:

emptiness of $\bigcap_{n} K_{n}$ is semi-decidable.

Proof. Because $X$ is compact by exhaustibility, $K_{n}$ is also compact as it is closed. Because $X$ is Hausdorff, $\bigcap_{n} K_{n}=\emptyset$ if and only if there is $n$ such that $K_{n}=\emptyset$. But emptiness of this set is decidable uniformly in $n$ by the algorithm $\forall x \in X . x \notin K_{n}$. Hence a semidecision procedure is given by $\exists n . \forall x \in X . x \notin K_{n}$. 
As a preparation for a lemma that will be applied to compute unique solutions, notice that if a singleton $\{u\} \subseteq \mathbb{N}^{Z}$ is exhaustible, then the function $u$ is computable, because $u(z)=\mu m . \forall v \in\{u\} \cdot v(z)=m$. Moreover, $u$ is computable uniformly in $\forall_{\{u\}}$, in the sense that there is a computable functional

$$
U: S \rightarrow \mathbb{N}^{Z} \quad \text { with } \quad S=\left\{\phi \in 2^{2^{\mathbb{N}} Z} \mid \phi=\forall_{\{v\}} \text { for some } v \in \mathbb{N}^{Z}\right\},
$$

such that $u=U\left(\forall_{\{u\}}\right)$, namely $U(\phi)(z)=\mu m \cdot \phi(\lambda u \cdot u(z)=m)$. Lemma 4.4 below generalizes this, using an argument from [6] that was originally used to prove that nonempty exhaustible subsets of Kleene-Kreisel spaces are computable images of the Cantor space and hence searchable. Here we find additional applications and further useful generalizations.

Lemma 4.4. Let $X$ be an exhaustible subspace of a Kleene-Kreisel space and $K_{n} \subseteq X$ be a sequence of sets that are exhaustible uniformly in $n$ and satisfy $K_{n} \supseteq K_{n+1}$. Then, uniformly in the data:

$$
\text { if } \bigcap_{n} K_{n} \text { is a singleton }\{x\} \text {, then } x \text { is computable. }
$$

Proof. By Lemma 3.2 $X$ is a computable retract of its Kleene-Kreisel super-space. Because any Kleene-Kreisel space is a computable retract of a Kleene-Kreisel space of the form $\mathbb{N}^{Z}$, and because retractions compose, there are computable maps $s: X \rightarrow \mathbb{N}^{Z}$ and $r: \mathbb{N}^{Z} \rightarrow X$ with $r \circ s=\operatorname{id}_{X}$. It suffices to show that the function $u=s(x) \in \mathbb{N}^{Z}$ is computable, because $x=r(u)$. The sets $L_{n}=s\left(K_{n}\right) \subseteq \mathbb{N}^{Z}$, being computable images of exhaustible sets, are themselves exhaustible. For any $z \in Z$, the set $U_{z}=\left\{v \in \mathbb{N}^{Z}\right.$ $v(z)=u(z)\}$ is clopen and $\bigcap_{n} L_{n}=\{u\} \subseteq U_{z}$. Because $\mathbb{N}^{Z}$ is Hausdorff, because $L_{n} \supseteq L_{n+1}$, because each $L_{n}$ is compact and because $U_{z}$ is open, there is $n$ such that $L_{n} \subseteq U_{z}$. That is, $v \in L_{n}$ implies $v(z)=u(z)$. Therefore, for every $z \in Z$ there is $n$ such that $v(z)=w(z)$ for all $v, w \in L_{n}$. Now, the map $n(z)=\mu n . \forall v, w \in L_{n} . v(z)=w(z)$ is computable by the exhaustibility of $L_{n}$. But $u \in L_{n(z)}$ for any $z \in Z$ and therefore $u$ is computable by exhaustibility as $u(z)=\mu m . \forall v \in L_{n(z)} \cdot v(z)=m$, as required.

To build sets $K_{n}$ suitable for applying these two lemmas, we use:

Lemma 4.5. For every computable retract of a Kleene-Kreisel space, there is a family $\left(=_{n}\right)$ of equivalence relations that are decidable uniformly in $n$ and satisfy

$$
\begin{aligned}
x=x^{\prime} & \Longleftrightarrow \quad \forall n . x={ }_{n} x^{\prime}, \\
x={ }_{n+1} x^{\prime} & \Longrightarrow \quad x={ }_{n} x^{\prime} .
\end{aligned}
$$

Proof. Let $X$ be a Kleene-Kreisel space and $s: X \rightarrow \mathbb{N}^{Z}$ and $r: \mathbb{N}^{Z} \rightarrow X$ be computable maps with $Z$ a Kleene-Kreisel space and $r \circ s=\operatorname{id}_{X}$. By the density theorem, there is a computable dense sequence $\delta_{n} \in Z$. Then the definition

$$
x={ }_{n} x^{\prime} \Longleftrightarrow \forall i<n . s(x)\left(\delta_{i}\right)=s\left(x^{\prime}\right)\left(\delta_{i}\right)
$$

clearly produces an equivalence relation that is decidable uniformly in $n$ and satisfies $x={ }_{n+1} x^{\prime} \Longrightarrow x={ }_{n} x^{\prime}$. Moreover, $x=x^{\prime}$ iff $s(x)=s\left(x^{\prime}\right)$, because $s$ is injective, iff $s(x)\left(\delta_{n}\right)=s\left(x^{\prime}\right)\left(\delta_{n}\right)$ for every $n$, by density, iff $x={ }_{n} x^{\prime}$ for every $n$, by definition.

Proof of Theorem 4.1. The set $K_{n}=\left\{x \in X \mid f(x)={ }_{n} y\right\}$, being a decidable subset of an exhaustible space, is exhaustible. Therefore the result follows from Lemmas 4.3 and 4.4 . because $x \in \bigcap_{n} K_{n}$ iff $f(x)={ }_{n} y$ for every $n$ iff $f(x)=y$ by Lemma 4.5

Algorithms 4.6. In summary, the algorithm for semi-deciding non-existence of solutions is

$$
\exists n . \forall x \in X . f(x) \neq_{n} y,
$$


and that for computing the solution $x_{0}$ as a function of $\forall_{X}, f$, and $y$ is:

$$
\begin{aligned}
\forall x \in K_{n} \cdot p(x) & =\forall x \in X \cdot f(x)={ }_{n} y \Longrightarrow p(x), \\
\forall v \in L_{n} \cdot q(v) & =\forall x \in K_{n} \cdot q(s(x)), \\
n(z) & =\mu n \cdot \forall v, w \in L_{n} \cdot v(z)=w(z), \\
u(z) & =\mu m \cdot \forall v \in L_{n(z)} \cdot v(z)=m, \\
x_{0} & =r(u) .
\end{aligned}
$$

Here $r: \mathbb{N}^{Z} \rightarrow X$ is a computable retraction with section $s: X \rightarrow \mathbb{N}^{Z}$, where $Z$ is a Kleene-Kreisel space, as constructed in the proof of Lemma 4.4

Of course, even in the absence of uniqueness, approximate solutions with precision $n$ are trivially computable with the algorithm

$$
\varepsilon_{X}\left(\lambda x . f(x)={ }_{n} y\right),
$$

using the fact that non-empty exhaustible subsets of Kleene-Kreisel spaces are searchable. But the above unique-solution algorithm uses the quantification functional $\forall x$ rather than the selection functional $\varepsilon_{X}$. In the next section we compute solutions as limits of approximate solutions.

\section{Equations over Metric Spaces}

For the purposes of this and the following section, we can work with computational spaces in the sense of TTE [13] using Baire-space representations, or equivalently, using partial equivalence relations on representatives living in effectively given domains [2]. Our development applies to both, and we can more generally assume for the former that representatives form subspaces of arbitrary Kleene-Kreisel spaces rather than just the Baire space $\mathbb{N}^{\mathbb{N}}$. We first formulate the main result of this section and then supply the missing notions in Definition 5.2.

Theorem 5.1. Let $X$ and $Y$ be computational metric spaces with $X$ computationally complete and having an exhaustible Kleene-Kreisel representation.

If $f: X \rightarrow Y$ is continuous and $y \in Y$, then, uniformly in $f, y$ and the exhaustibility condition:

1. It is semi-decidable whether the equation $f(x)=y$ fails to have a solution.

2. If $f(x)=y$ has a unique solution $x \in X$, then it is computable.

Hence any computable bijection $f: X \rightarrow Y$ has a computable inverse, uniformly in $f$ and the exhaustibility condition.

Given that exhaustibility is a computational counter-part of the topological notion of compactness, and that compact metric spaces are complete, it is natural to conjecture that, at least under suitable computational conditions, the assumption of computational completeness in the above theorem is superfluous. We leave this as an open question. In connection with this, notice that this theorem is analogous to a well-known result in constructive mathematics [4], with the assumptions reformulated in our higher-type computational setting.

There is a technical difficulty in the proof of the theorem: at the intensional level, where computations take place, solutions are unique only up to equivalence of representatives. In order to overcome this, we work with pseudo-metric spaces at the intensional level and 
with a notion of decidable closeness for them. Recall that a pseudo-metric on a set $X$ is a function $d: X \times X \rightarrow[0, \infty)$ such that

$$
d(x, x)=0, \quad d(x, y)=d(y, x), \quad d(x, z) \leq d(x, y)+d(y, z) .
$$

Then $d$ is a metric if it additionally satisfies $d(x, y)=0 \Longrightarrow x=y$. If $d$ is only a pseudo-metric, then $(\sim)$ defined by

$$
x \sim y \Longleftrightarrow d(x, y)=0
$$

is an equivalence relation, referred to as pseudo-metric equivalence. A pseudo-metric topology is Hausdorff if and only if it is $T_{0}$ if and only if the pseudo-metric is a metric. Moreover, two points are equivalent if and only if they have the same neighbourhoods. Hence any sequence has at most one limit up to equivalence.

A computational metric space is a computational pseudo-metric space in which the pseudo-metric is actually a metric, and hence we formulate the following definitions in the generality of pseudo-metric spaces.

Definition 5.2. We work with any standard admissible representation of the Hausdorff space $[0, \infty)$.

1. A computational pseudo-metric space is a computational space $X$ endowed with a computable pseudo-metric, denoted by $d=d_{X}: X \times X \rightarrow[0, \infty)$.

2. A fast Cauchy sequence in a computational pseudo-metric space $X$ is a sequence $x_{n} \in X$ with $d\left(x_{n}, x_{n+1}\right)<2^{-n}$. The subspace of $X^{\mathbb{N}}$ consisting of fast Cauchy sequences is denoted by $\operatorname{Cauchy}(X)$.

3. A computational pseudo-metric space $X$ is called computationally complete if every sequence $x_{n} \in \operatorname{Cauchy}(X)$ has a limit uniformly in $x_{n}$.

4. A computational pseudo-metric space $X$ has decidable closeness if there is a family of relations $\sim_{n}$ on $X$ that are decidable uniformly in $n$ and satisfy:
(a) $x \sim_{n} y \Longrightarrow d(x, y)<2^{-n}$,
(b) $x \sim y \Longrightarrow \forall_{n} \cdot x \sim_{n} y$.
(c) $x \sim_{n+1} y \Longrightarrow x \sim_{n} y$,
(d) $x \sim_{n} y \Longleftrightarrow y \sim_{n} x$,
(e) $x \sim_{n+1} y \sim_{n+1} z \Longrightarrow x \sim_{n} z$.

The last condition is a counter-part of the triangle inequality. It follows from the first condition that if $x \sim_{n} y$ for every $n$, then $x \sim y$. Write

$$
[x]=\{y \in X \mid x \sim y\}, \quad[x]_{n}=\left\{y \in X \mid x \sim_{n} y\right\} .
$$

Then the equivalence class $[x]$ is the closed ball of radius 0 centered at $x$.

For instance, the spaces $\mathbb{R}$ and $[0, \infty)$ are computationally complete metric spaces under the Euclidean metric, but don't have decidable closeness.

We are now ready to prove the theorem.

Lemma 5.3. For every computational metric space $X$ there is a canonical computable pseudo-metric $d=d\ulcorner X\urcorner$ on the representing space $\ulcorner X\urcorner$ such that:

1. The representation map $\rho=\rho_{X}:\ulcorner X\urcorner \rightarrow X$ is an isometry:

$$
d(t, u)=d(\rho(t), \rho(u)) .
$$

In particular: 
(a) $t \sim u \Longleftrightarrow d(t, u)=0 \Longleftrightarrow \rho(t)=\rho(u)$.

(b) If $f: X \rightarrow Y$ is a computable map of metric spaces, then any representative $\ulcorner f\urcorner:\ulcorner X\urcorner \rightarrow\ulcorner Y\urcorner$ preserves the relation $(\sim)$.

2. If $X$ is computationally complete, then so is $\ulcorner X\urcorner$.

3. The representing space $\ulcorner X\urcorner$ has decidable closeness.

Proof. Construct $d\ulcorner X\urcorner:\ulcorner X\urcorner \times\ulcorner X\urcorner \rightarrow[0, \infty)$ as the composition of a computable representative $\left\ulcorner d_{X}\right\urcorner:\ulcorner X\urcorner \times\ulcorner X\urcorner \rightarrow\ulcorner[0, \infty)\urcorner$ of $d_{X}: X \times X \rightarrow[0, \infty)$ with the representation map $\rho_{[0, \infty)}:\ulcorner[0, \infty)\urcorner \rightarrow[0, \infty)$. A limit operator for $\ulcorner X\urcorner$ from a limit operator for $X$ is constructed in a similar manner. For given $t, u \in\ulcorner X\urcorner$, let $q_{n}$ be the $n$-th term of the sequence $\left\ulcorner d_{X}\right\urcorner(t, u) \in\ulcorner[0, \infty)\urcorner \subseteq \operatorname{Cauchy}(\mathbb{Q})$, and define $t \sim_{n} u$ to mean that $\left[-2^{-n}, 2^{-n}\right] \subseteq\left[q_{n}-2^{-n+1}, q_{n}+2^{-n+1}\right]$.

Lemma 5.4. Let $Z$ be a subspace of a Kleene-Kreisel space with complete computational pseudo-metric structure and decidable closeness, and $K_{n} \subseteq Z$ be a sequence of sets that are exhaustible uniformly in $n$ and satisfy $K_{n} \supseteq K_{n+1}$. Then, uniformly in the data:

if $\bigcap_{n} K_{n}$ is an equivalence class, then it has a computable member.

Proof. Let $z \in \bigcap_{n} K_{n}$. For any $m$, we have $\bigcap_{n} K_{n}=[z] \subseteq[z]_{m+1}$, and hence there is $n$ such that $K_{n} \subseteq[z]_{m+1}$, because the sets $K_{n}$ are compact, because $K_{n} \supseteq K_{n+1}$, because $Z$ is Hausdorff and because $[z]_{m+1}$ is open. Hence for every $u \in K_{n}$ we have $u \sim_{m+1} z$, and so for all $u, v \in K_{n}$ we have $u \sim_{m} v$. By the exhaustibility of $K_{n}$ and the decidability of $\left(\sim_{n}\right)$, the function $n(m)=\mu n . \forall u, v \in K_{n} . u \sim_{m} v$ is computable. By the searchability of $K_{n}$, there is a computable sequence $u_{m} \in K_{n(m)}$. Because $n(m) \leq n(m+1)$, we have that $K_{n(m)} \supseteq K_{n(m+1)}$ and hence $u_{m} \sim_{m} u_{m+1}$ and so $d\left(u_{m}, u_{m+1}\right)<2^{-m}$ and $u_{m}$ is a Cauchy sequence. By completeness, $u_{m}$ converges to a computable point $u_{\infty}$. Because $z \in K_{n(m)}$, we have $u_{m} \sim_{m} z$ for every $m$, and hence $d\left(u_{m}, z\right)<2^{-m}$. And because $d\left(u_{\infty}, u_{m}\right)<2^{-m+1}$, the triangle inequality gives $d\left(u_{\infty}, z\right)<2^{-m}+2^{-m+1}$ for every $m$ and hence $d\left(u_{\infty}, z\right)=0$ and therefore $u_{\infty} \in \bigcap_{n} K_{n}$.

The proof of the following is essentially the same as that of Theorem 4.1, but uses Lemma 5.4 rather than Lemma 4.4, and Lemma 5.3 instead of Lemma 4.5 .

Lemma 5.5. Let $Z$ and $W$ be subspaces of Kleene-Kreisel spaces with computational pseudo-metric structure and decidable closeness, and assume that $Z$ is computationally complete and exhaustible.

If $g: Z \rightarrow W$ is a computable map that preserves pseudo-metric equivalence and $w \in W$ is computable, then, uniformly in $\forall_{Z}, g$, and $w$ :

1. It is semi-decidable whether the equivalence $g(z) \sim w$ fails to have a solution $z \in Z$.

2. If $g(z) \sim w$ has a unique solution $z \in Z$ up to equivalence, then some solution is computable.

Proof. The set $K_{n}=\left\{z \in Z \mid g(z) \sim_{n} w\right\}$, being a decidable subset of an exhaustible space, is exhaustible. Therefore the result follows from Lemmas 4.3 and 5.4, because $z \in \bigcap_{n} K_{n}$ iff $g(z) \sim_{n} w$ for every $n$ iff $g(z)=w$.

Algorithm 5.6. The solution $z=u_{\infty}$ is then computed from $\forall_{Z}, g$ and $w$ as follows, where we have expanded $\forall_{K_{n}}$ as a quantification over $Z$ :

$$
\begin{aligned}
n(m) & =\mu n . \forall u, v \in Z . g(u) \sim_{n} w \wedge g(v) \sim_{n} w \Longrightarrow u \sim_{m} v, \\
u_{\infty} & =\lim _{m} \varepsilon_{K}\left(\lambda z \cdot g(z) \sim_{n(m)} w\right) .
\end{aligned}
$$


Thus, although there are common ingredients with Theorem 4.1 , the resulting algorithm is different from 4.6 because it relies on the limit operator and approximate solutions.

But, for Theorem 5.1, approximate solutions are computable uniformly in $\ulcorner f\urcorner$ and $\ulcorner y\urcorner$ only, as different approximate solutions are obtained for different representatives of $f$ and $y$ :

Proof of Theorem 5.1 Let $f: X \rightarrow Y$ and $y \in Y$ be computable. Now apply Lemma 5.5 with $Z=\ulcorner X\urcorner, W=\ulcorner Y\urcorner, g=\ulcorner f\urcorner, w=\ulcorner y\urcorner$, using Lemma 5.3 to fulfil the necessary hypotheses. If $f(x)=y$ has a unique solution $x$, then $g(z) \sim w$ has a unique solution $z$ up to equivalence, and $x=\rho(z)$ for any solution $z$, and hence $x$ is computable. Because $g$ preserves $(\sim)$ by Lemma 5.3 if $g(z) \sim w$ has a solution $z$, then $x=\rho(z)$ is a solution of $f(x)=y$. This shows that $f(x)=y$ has a solution if and only if $g(z)=w$ has a solution, and we can reduce the semi-decision of absence of solutions of $f(x)=y$ to absence of solutions of $g(z)=w$.

Before giving examples in computational real analysis, in Section 6, we clarify some aspects of the above development.

Remark 5.7. In the above definition, we don't require the representation topology of $X$ to agree with the pseudo-metric topology generated by open balls. But notice that the metric topology is always coarser than the representation topology, because, by continuity of the metric, open balls are open in the representation topology. Hence the representation topology of any computational metric space is Hausdorff. Moreover, if $X$ has an exhaustible Kleene-Kreisel space of representatives and the metric topology is compact, then both topologies agree, because no compact Hausdorff topology can be properly refined to another compact Hausdorff topology.

Recall that an ultra-metric space is a metric space for which the triangle inequality holds in the stronger form $d(x, z) \leq \max (d(x, y), d(y, z))$, and that an ultra-metric topology is zero-dimensional because open balls are closed. Recall the equivalence relations $\left(={ }_{n}\right)$ given in Lemma 4.5

Proposition 5.8. Any space $X$ that is a computable restract of some Kleene-Kreisel space becomes a computational ultra-metric space when it is equipped with the metric d defined by

$$
d(x, y)=\inf \left\{2^{-n} \mid x=_{n} y\right\} .
$$

Moreover:

1. The metric has decidable closeness given by $\left(\sim_{n}\right)=\left(=_{n}\right)$.

2. The metric topology is in general strictly coarser than the computational topology, but both agree on compact subsets.

3. Exhaustible subspaces with the relative metric are computationally complete.

Proof. Computability of the ultra-metric and decidability of closeness are easy.

Let $s: X \rightarrow \mathbb{N}^{Z}$ and $r: \mathbb{N}^{Z} \rightarrow X$ be the same computable functions selected in the proof of Lemma 4.5 , let $\delta$ be the same dense sequence, and for $u, v \in \mathbb{N}^{Z}$ define $u={ }_{n} v \Longleftrightarrow \forall i<n . u\left(\delta_{i}\right)=v\left(\delta_{i}\right)$ so that $x={ }_{n} y$ iff $s(x)={ }_{n} s(y)$.

Agreement of topologies: A subbasic open set in the topology of pointwise convergence of $\mathbb{N}^{Z}$ is of the form $N(z, V)=\left\{u \in \mathbb{N}^{Z} \mid u(z) \in V\right\}$ with $z \in Z$ and $V \subseteq \mathbb{N}$. Now $u={ }_{n} v$ iff $d(u, v)<2^{-n}$, and hence the open ball $B_{2^{-n}}(u)$ is the intersection of the pointwise open sets $N\left(\delta_{i},\left\{u\left(\delta_{i}\right)\right\}\right)$, for $i<n$, and hence open balls are open in the topology of pointwise convergence. For a compact subspace of $\mathbb{N}^{Z}$, density of $\delta$ gives that metric topology agrees with the pointwise topology. But the relative topology on compact subsets of $\mathbb{N}^{Z}$ coincides with the topology of pointwise convergence, by classical, and easy, Arzela-Ascoli type arguments, and hence the three topologies agree. The reduction of this to $X$ via the retraction is easy. 
Completeness: Let $z_{n} \in Z$ be a fast Cauchy sequence. Then $z_{n}=_{n} z_{n+1}$ and hence $s\left(z_{n}\right)={ }_{n} s\left(z_{n+1}\right)$. It suffices to show that the sequence $f_{n}=s\left(z_{n}\right)$ converges to a computable limit $f_{\infty}$, because then the sequence $z_{n}=r\left(f_{n}\right)$ converges to the computable point $z_{\infty}=r\left(f_{\infty}\right)$ by continuity of $r$. The set $L=s(K)$ is exhaustible because it is a computable image of an exhaustible set. For any $n$, the set $L_{n}=\left\{g \in L \mid g={ }_{n} f_{n}\right\}$ is exhaustible because it is a decidable subset of an exhaustible set, and $f_{n} \in L_{n}$. By compactness, $\bigcap_{n} L_{n} \neq \emptyset$ because clearly $L_{n} \supseteq L_{n+1}$. If $g, h \in \bigcap_{n} L_{n}$, then $g={ }_{n}$ $f_{n}={ }_{n} h$ for every $n$, and hence $g=h$, and so $\bigcap_{n} L_{n}=\left\{f_{\infty}\right\}$ for some computable $f_{\infty}$ by Lemma 4.4 Because $f_{\infty} \in \bigcap_{n} L_{n}$, we have $f_{\infty}={ }_{n} f_{n}$ for every $n$. Hence if some ball $B_{2^{-k}}(h)$ is a neighbourhood of $f_{\infty}$, then $h={ }_{k} f_{\infty}={ }_{k} f_{n}$ for all $n \geq k$, and hence $f_{n} \in B_{k}(h)$ for all $n \geq k$, which shows that $f_{n} \rightarrow f_{\infty}$.

In view of this proposition, Lemma 5.4 generalizes Lemma 4.4 But Lemma 4.4 cannot be eliminated, because it is used to prove the proposition.

Algorithm 5.9. Expanding Lemma 4.4, the algorithm for computing $\lim _{n} f_{n}$ for a fast Cauchy sequence $f_{n} \in L \subseteq \mathbb{N}^{Z}$ with $L$ exhaustible is:

$$
\begin{aligned}
n(z) & =\mu n . \forall g, h \in L . g={ }_{n} f_{n} \wedge h={ }_{n} f_{n} \Longrightarrow g(z)=h(z), \\
\lim _{n} f_{n} & =\lambda z \cdot \mu m . \forall g \in L . g={ }_{n(z)} f_{n(z)} \Longrightarrow g(z)=m .
\end{aligned}
$$

Notice that "fast" amounts to $f_{n}={ }_{n} f_{n+1}$.

Remark 5.10. Independently of this, Matthias Schröder (personal communication) showed that if a QCB space $X$ is the sequential coreflection of a zero-dimensional topology, then there is a metric $d$ on $X$ such that: (1) The topology induced by $d$ is coarser than that of $X$ and than the zero-dimensional topology. (2) On compact subsets $X$, the three topologies agree. (3) The image of $d$ is $\{0\} \cup\left\{2^{-n} \mid n \in \mathbb{N}\right\}$. This applies to all retracts of KleeneKreisel spaces in particular, as their topologies satisfy the hypotheses. His construction uses countable pseudo-bases rather than dense sequences. However, computational versions of these statements remain to be considered.

\section{Exhaustible Spaces of Analytic Functions}

For any $\epsilon \in(0,1)$, any $x \in[-\epsilon, \epsilon]$, any $b>0$, and any sequence $a \in[-b, b]^{\mathbb{N}}$, the Taylor series $\sum_{n} a_{n} x^{n}$ converges to a number in the interval $[-b /(1+\epsilon), b /(1-\epsilon)]$. The following is proved by a standard computational analysis argument:

Lemma 6.1. Any analytic function $f \in \mathbb{R}^{[-\epsilon, \epsilon]}$ of the form $f(x)=\sum_{n} a_{n} x^{n}$ is computable uniformly in any given $\epsilon \in(0,1), b>0$ and $a \in[-b, b]^{\mathbb{N}}$.

Definition 6.2. Denote by $A=A(\epsilon, b) \subseteq \mathbb{R}^{[-\epsilon, \epsilon]}$ the subspace of such analytic functions and by $T=T_{\epsilon, b}:[-b, b]^{\mathbb{N}} \rightarrow A(\epsilon, b)$ the functional that implements the uniformity condition, so that $f=T(a)$.

The following results also hold uniformly in $\epsilon$ and $b$, but we omit explicit indications for the sake of brevity. The results are uniform in the exhaustibility assumptions too. Because $[-b, b]^{\mathbb{N}}$ is compact and $T$ is continuous, the space $A$ is compact as well. Moreover:

Theorem 6.3. The space A has an exhaustible set of Kleene-Kreisel representatives.

Proof. The space $[-b, b]^{\mathbb{N}}$ has an exhaustible space of representatives, e.g. using signeddigit binary representation. Because exhaustible spaces are preserved by computable images, the image of any representative $\ulcorner T\urcorner:\left\ulcorner[-b, b]^{\mathbb{N}}\right\urcorner \rightarrow\ulcorner A\urcorner$ of $T$ gives an exhaustible set of representatives of $A$ contained in the set $\ulcorner A\urcorner$ of all representatives of $A$. 
Hence the solution of a functional equation with a unique analytic unknown in $A$ can be computed using Theorem 5.1 .

Lemma 6.4. For any non-empty space $X$ with an exhaustible set of Kleene-Kreisel representatives, the maximum- and minimum-value functionals

$$
\max _{X}, \min _{X}: \mathbb{R}^{X} \rightarrow \mathbb{R}
$$

are computable.

Of course, any $f \in \mathbb{R}^{X}$ attains its maximum value because it is continuous and because spaces with exhaustible sets of representatives are compact.

Proof. We discuss max only. By e.g. the algorithm given in [12], this is the case for $X=2^{\mathbb{N}}$. Because the representing space $\ulcorner X\urcorner$, being a non-empty exhaustible subspace of a Kleene-Kreisel space, is a computable image of the Cantor space, the space $X$ itself is a computable image of the cantor space, say with $q: 2^{\mathbb{N}} \rightarrow X$. Then the algorithm $\max _{X}(f)=\max _{2^{N}}(f \circ q)$ gives the required conclusion.

Corollary 6.5. If $K$ is a subspace of a metric space $X$ and $K$ has an exhaustible set of Kleene-Kreisel representatives, then $K$ is computably located in $X$, in the sense that the distance function $d_{K}: X \rightarrow \mathbb{R}$ defined by

$$
d_{K}(x)=\min \{d(x, y) \mid y \in K\}
$$

is computable.

Corollary 6.6. For any metric space $X$ with an exhaustible set of Kleene-Kreisel representatives, the max-metric $d(f, g)=\max \{d(f(x), g(x)) \mid x \in X\}$ on $\mathbb{R}^{X}$ is computable.

Corollary 6.7. For $f \in \mathbb{R}^{[-\epsilon, \epsilon]}$, it is semi-decidable whether $f \notin A$.

Proof. Because $A$ is computationally located in $\mathbb{R}^{[-\epsilon, \epsilon]}$ as it has an exhaustible set of representatives, and because $f \notin A \Longleftrightarrow d_{A}(f) \neq 0$.

Another proof, which doesn't rely on the exhaustibility of a set of representatives of $A$, uses Theorem 5.1 $f \notin A$ if and only if the equation $T(a)=f$ doesn't have a solution $a \in$ $[-b, b]^{\mathbb{N}}$. But this alternative proof relies on a complete metric on $[-b, b]^{\mathbb{N}}$. For simplicity, we consider a standard construction for 1-bounded metric spaces. Because we apply this to metric spaces with exhaustible sets of representatives, this is no loss of generality as the diameter of such a space is computable as $\max (\lambda x \cdot \max (\lambda y . d(x, y)))$ and hence the metric can be computably rescaled to become 1-bounded.

Lemma 6.8. For any computational 1 -bounded metric space $X$, the metric on $X^{\mathbb{N}}$ defined by $d(x, y)=\sum_{n} 2^{-n-1} d\left(x_{n}, y_{n}\right)$ is computable and 1-bounded, and it is computationally complete if $X$ is.

Proof. Use the fact that the map $[0,1]^{\mathbb{N}} \rightarrow[0,1]$ that sends a sequence $a \in[0,1]^{\mathbb{N}}$ to the number $\sum_{n} 2^{-n-1} a_{n}$ is computable. Regarding completeness, it is well known that a sequence in the space $X^{\mathbb{N}}$ is Cauchy if and only if it is componentwise Cauchy in $X$, and in this case its limit is calculated componentwise.

Corollary 6.9. The Taylor coefficients of any $f \in$ A can be computed from $f$.

Proof. Because $[-b, b]^{\mathbb{N}}$ has an exhaustible set of representatives, the function $T$ has a computable inverse by Theorem 5.1 and Lemma 6.8 . 


\section{References}

[1] I. Battenfeld, M. Schröder, and A. Simpson. A convenient category of domains. In Computation, meaning, and logic: articles dedicated to Gordon Plotkin, volume 172 of Electron. Notes Theor. Comput. Sci., pages 69-99. Elsevier, Amsterdam, 2007.

[2] A. Bauer. A relationship between equilogical spaces and type two effectivity. $M L Q$ Math. Log. Q., 48(suppl. 1):1-15, 2002.

[3] M.J. Beeson. Foundations of Constructive Mathematics. Springer, 1985.

[4] E. Bishop and D. Bridges. Constructive Analysis. Springer, Berlin, 1985.

[5] Normann D. Applications of the kleenekreisel density theorem to theoretical computer science. In B. Cooper S.B., L we and Sorbi A., editors, New Computational Paradigms, pages 119-138. Springer, 2007.

[6] M. Escardó. Exhaustible sets in higher-type computation. Log. Methods Comput. Sci., 4(3):3:3, 37, 2008.

[7] M.H. Escardó, J. Lawson, and A. Simpson. Comparing Cartesian closed categories of (core) compactly generated spaces. Topology Appl., 143(1-3):105-145, 2004.

[8] D. Normann. Recursion on the countable functionals, volume 811 of Lec. Not. Math. Springer, 1980.

[9] D. Normann. The continuous functionals. In Handbook of computability theory, volume 140 of Stud. Logic Found. Math., pages 251-275. North-Holland, Amsterdam, 1999.

[10] D. Normann. Computing with functionals - computability theory or computer science? Bull. Symbolic Logic, 12(1):43-59, 2006.

[11] M. Schröder. Extended admissibility. Theoret. Comput. Sci., 284(2):519-538, 2002.

[12] A. Simpson. Lazy functional algorithms for exact real functionals. Lec. Not. Comput. Sci., 1450:323-342, 1998.

[13] K. Weihrauch. Computability. Springer, 1987. 